\title{
Effects on patient care of introducing prehospital intravenous nalbuphine hydrochloride
}

\author{
P Hyland-McGuire, H R Guly
}

\begin{abstract}
Objective-Since March 1992, intravenous nalbuphine hydrochloride has been used prehospital by paramedics in the Plymouth area. This study assesses the impact of this intervention.

Methods-A prospective study of the parenteral analgesic requirements of 1000 consecutive patients arriving by ambulance at the accident and emergency (A\&E) department of a large district general hospital. Where parenteral analgesia was given in the $A \& E$ department but not by ambulance personnel, a questionnaire was sent to the ambulance crew concerned to ascertain the reasons for not having given nalbuphine.

Results-Of 1000 consecutive patients arriving by ambulance, $87(8.7 \%)$ had been given parenteral analgesia either prehospital, in A\&E, or in both places. Seventy five $(7.5 \%)$ needed parenteral analgesia in the A\&E department, 29 (2.9\%) had been given prehospital intravenous analgesia by paramedics, and a further seven $(0.7 \%)$ had been given parenteral analgesia by a general practitioner (GP). Thus 36 (3.6\%) received prehospital analgesia. Ten patients who had been given analgesia by paramedics required no further analgesia in $A \& E$, whereas 51 patients who had not been given prehospital analgesia required parenteral analgesia in the $A \& E$ department.
\end{abstract}

Conclusions-The introduction of nalbuphine for use by paramedics in prehospital care has increased prehospital parenteral analgesia from $1 \%$ in 1992 (given by GPs only) to $3.6 \%$ in the current study group, and $41 \%$ of patients requiring parenteral analgesia received analgesia prehospital. There may be further scope for extending the indications for nalbuphine use by ambulance personnel. (F Accid Emerg Med 1998;15:99-101)

Keywords: prehospital analgesia; paramedic; nalbuphine

The relief of pain is one of the priorities of emergency treatment. Intravenous nalbuphine hydrochloride given by paramedics prehospital is safe and effective. ${ }^{1}$
In March $1992,{ }^{2}$ a policy decision was made to allow the administration of nalbuphine hydrochloride by paramedics in the Plymouth division of the Devon Ambulance Service (now part of the Westcountry Ambulance Service Trust) in doses of $4 \mathrm{mg} / \mathrm{min}$, titrated according to response to a maximum of $30 \mathrm{mg}$ in patients with isolated limb fractures, burns, or suspected myocardial infarction who did not have contraindications to its use. These contraindications were: known hypersensivity to opioids, pregnancy, monoamine oxidase inhibitor treatment, and clinical contraindications such as head, chest, or abdominal injuries and hypotension. The maximum dose in the guidelines has since been reduced to $20 \mathrm{mg}$, but this can be exceeded following radio consultation with an experienced $A \& E$ doctor. A survey ${ }^{3}$ set in our accident and emergency (A\&E) department in 1991 before the introduction of nalbuphine use by paramedics showed that 69 patients from a series of 502 ambulance patients $(14 \%)$ received opiate analgesia in the A\&E department. Unpublished data from the same study showed that $1 \%$ of ambulance patients had been given prehospital analgesia by a general practitioner. Since then paramedics throughout the Westcountry Ambulance Service Trust have been giving nalbuphine prehospital and our present study assesses the impact this has had on patient care.

\section{Methods}

We analysed the parenteral analgesic requirement of 1000 consecutive patients arriving by ambulance at the $A \& E$ department of a large district general hospital (approximately 67000 new attendances per year) with a mixed urban and rural catchment area. A list of the previous days' ambulance attendances was generated on a daily basis from the $A \& E$ administration computer and the ambulance report forms and casualty cards were retrieved. A proforma was completed for each patient arriving by ambulance who had received parenteral analgesia, either prehospital or in $A \& E$, and the data were entered onto a spreadsheet. Patients who had not had parenteral analgesia were merely counted and the study was terminated at 27 days when the 1000th consecutive ambulance arrival was reached.

In cases where parenteral analgesia had been given in the $\mathrm{A} \& \mathrm{E}$ department but not by the 
Table 1 Conditions treated with nalbuphine by paramedics

Conditions treated with paramedic analgesia only (10) Chest pain

Fractures

Dislocations

Soft tissue injuries

Conditions treated by a paramedic but requiring further analgesia in $A \& E$ (19)

Chest pain

Fractures

Dislocations

Multiple injury

Amputation thumb

Soft tissue injury

ambulance personnel, a questionnaire was sent to the ambulance station for distribution to the relevant crew to determine the reasons for this. The results of these questionnaires were collated anonymously.

\section{Results}

AMBULANCE ARRIVALS SURVEY

Of 1000 consecutive patients arriving by ambulance over approximately 27 days, 87 $(8.7 \%)$ had been given parenteral analgesia either prehospital, in the A\&E department, or both. Seven patients $(0.7 \%)$ arriving by ambulance had been given parenteral analgesia by a general practitioner, and in two instances nalbuphine carried by the ambulance service was used. The indications were fractures or dislocations (5) and soft tissue injuries (2), and two patients required no further analgesia in the $A \& E$ department.

Twenty nine patients $(2.9 \%)$ arriving by ambulance had been given intravenous nalbuphine prehospital by paramedics. The total dose used was $20 \mathrm{mg}$ in 17 patients, $10 \mathrm{mg}$ in seven, and between 4 and $15 \mathrm{mg}$ in the other five. No adverse effects were noted in the ambulance report forms following nalbuphine use. Ten patients who had been given nalbuphine by paramedics did not require further parenteral analgesia in the $A \& E$ department. Nineteen patients required further analgesia in $\mathrm{A} \& \mathrm{E}$. Thus over one third of patients given analgesia by paramedics needed no further analgesia in the A\&E department. Table 1 shows the range of presenting complaints in these two groups. The list includes dislocations and soft tissue injuries which may be difficult to differentiate in the field from fractures, and a small number of conditions where nalbuphine was given outside the protocol following consultation with a senior $\mathrm{A} \& \mathrm{E}$ doctor.

In the $A \& E$ department, 75 patients $(7.5 \%)$ were given parenteral analgesia and 51 of these had not received prehospital analgesia. The most commonly used preparation was morphine ( 51 patients), with 10 patients receiving diamorphine, nine receiving pethidine, and
Table 2 Initial diagnoses of patients receiving analgesia

\begin{tabular}{lcc}
\hline Trauma & & Total \\
\hline Isolated limb fracture & 30 \\
$\quad$ Fracture neck of femur & 14 & \\
$\quad$ Others & 16 & 12 \\
Dislocations & 6 & \\
$\quad$ Total hip replacement & 2 & \\
Ankle & 1 & \\
Shoulder & 1 & \\
Elbow & 1 & \\
Metacarpals & 1 & \\
DHS loosening & & 7 \\
Multiple injuries & & 8 \\
Other fractures & & 7 \\
Soft tissue injuries & & 4 \\
Extensive lacerations & & 1 \\
Amputations & & 11 \\
Non-trauma & & 3 \\
Chest pain & & 1 \\
Back pain & & 1 \\
Renal colic & & 1 \\
Pancreatitis & & 1 \\
Testicular torsion & & \\
Ectopic pregnancy & & \\
\hline
\end{tabular}

DHS, dynamic hip screw

four receiving intramuscular diclofenac. One patient received both pethidine and morphine in the department.

Of the 87 patients receiving analgesia, 69 attended following trauma and 18 following non-traumatic episodes. Table 2 illustrates the presenting complaints of these two groups.

\section{QUESTIONNAIRE}

Fifty one questionnaires were sent to ambulance crews in situations where parenteral analgesia was given in the $\mathrm{A} \& \mathrm{E}$ department but not by paramedics. Thirty six completed forms were returned (a $71 \%$ response rate). The reasons cited for not having given nalbuphine are shown in table 3.

\section{Discussion}

This study was undertaken to close the audit loop initiated by Chambers and Guly. ${ }^{3}$ Unpublished data from that study based in the same $\mathrm{A} \& \mathrm{E}$ department showed that before the introduction of nalbuphine for use by paramedics, five patients out of 502 consecutive ambulance arrivals ( $1 \%$ ) had received prehospital analgesia (invariably given by a general practitioner) and $69(14 \%)$ had been given analgesia in A\&E. The increase from $5 / 502$ to $36 / 1000$ in patients receiving prehospital analgesia is statistically significant $\left(\chi^{2}=8.5352, \mathrm{p}=\right.$ $0.003)$. This change meant that $41 \%$ of patients requiring parenteral analgesia received it prehospital by paramedics.

We were surprised that the requirement for parenteral analgesia has decreased since 1991 $(8.7 \% v 14 \%)$ at a time when there has been an increased emphasis on the need for adequate and timely analgesia. The decrease from

Table 3 Reasons for not having given nalbuphine

\begin{tabular}{|c|c|c|c|c|c|}
\hline Paramedic factors & & Patient factors & & Outside protocol & \\
\hline $\begin{array}{l}\text { Not qualified to give nalbuphine } \\
\text { Cannot remember patient } \\
\text { Transported to hospital as priority }\end{array}$ & $\begin{array}{l}3 \\
5 \\
2\end{array}$ & $\begin{array}{l}\text { Patient said not in pain } \\
\text { Entonox sufficed for analgesia } \\
\text { Nalbuphine offered but refused } \\
\text { Positioning relieved pain } \\
\text { Saline flush relieved pain }\end{array}$ & $\begin{array}{l}6 \\
3 \\
3 \\
1 \\
1\end{array}$ & $\begin{array}{l}\text { Head injury } \\
\text { Abdominal injury } \\
\text { Multiple injury } \\
\text { Ectopic pregnancy } \\
\text { Back pain } \\
\text { Patient too young }\end{array}$ & $\begin{array}{l}3 \\
2 \\
1 \\
1 \\
3 \\
3\end{array}$ \\
\hline
\end{tabular}


69:433 in 1991 to $87: 917$ in 1996 is statistically significant $\left(\chi^{2}=9.1397, \mathrm{p}=0.003\right)$. This almost certainly represents an increased use of the ambulance service by patients with less acute conditions over the five year period. Emergency ambulance calls in the catchment area have shown an average increase of $6.7 \%$ each year over the same period (Westcountry Ambulance Trust Service statistics).

If the administration of parenteral analgesia by doctors in the $A \& E$ department is taken as defining those patients who need analgesia, there has been an increase in the proportion given analgesia prehospital from five of 69 $(7.25 \%)$ in 1991 to 36 of $87(41 \%)$ in the current study. Table 3 shows that there may be scope for further improvement if the indications for nalbuphine were extended (13 fell outside the protocol) and all paramedic crews were fully trained to give nalbuphine (three were not qualified to give nalbuphine).

Sixty seven per cent of patients given prehospital analgesia required further parenteral analgesia in the $A \& E$ department. This was not entirely unexpected, as repeat doses of analgesia would often be given for many of the conditions encountered. Fourteen patients who received the maximum dose of 20 $\mathrm{mg}$ required further analgesia in the $\mathrm{A} \& \mathrm{E}$ department. These figures suggest that more patients might benefit from nalbuphine and a maximum dose of $30 \mathrm{mg}$ rather than $20 \mathrm{mg}$ might be more appropriate. Since completion of this study, doses of $30 \mathrm{mg}$ have been given on several occasions by paramedics without any complications, following telephone consultation with a senior $A \& E$ doctor. Paramedics have been fully trained in the use of naloxone for reversal of respiratory depression caused by nalbuphine. Any increased use of nalbuphine must also be balanced against the increased on-scene time, with other implications on patient care. ${ }^{4}$

The questionnaire highlighted various points. Not all ambulance staff are currently qualified to give nalbuphine. Patients may not initially complain of pain to the ambulance personnel or may refuse opioids and opt for entonox, which may provide sufficient analgesia. Nalbuphine is currently given for a relatively narrow spectrum of conditions, and contraindications for its use featured prominently in the reasons for not giving it. There may be scope for extending its use for non-traumatic conditions such as back pain, renal colic, and abdominal pain after telephone advice from an experienced $A \& E$ doctor, and also in a few stable patients with more than one injury.

1 Stene JK, Stofberg L, MacDonald G, Myers RA, Ramsy A, Burns B. Nalbuphine analgesia in the prehospital setting.

2 Chambers JA, Guly HR. Prehospital intravenous nalbuphine administered by paramedics. Resuscitation 1994;27: 153-8.

3 Chambers JA, Guly HR. The need for better pre-hospital analgesia. Arch Emerg Med 1993;10:187-92

4 Johnson GS, Guly HR. The effect of pre-hospital administration of intravenous nalbuphine on on-scene times. J Accid Emerg Med 1995;12:20-2.

\section{Faculty of Accident and Emergency Medicine}

\section{Exit examinations}

The next Diet of the Faculty's Exit Examination will be on 6/7 May 1998 at the University of Edinburgh.

Inquiries to the Faculty of Accident and Emergency Medicine, 35-43 Lincoln's Inn Fields, London WC2A 3PN; tel +44 (0)171 4057071. 